\title{
Fault Tolerant Control Design for the Longitudinal Aircraft Dynamics using Quantitative Feedback Theory
}

\author{
Daniel Ossmann* \\ Institute of System Dynamics and Control \\ German Aerospace Center (DLR), 82234 Wessling, Germany
}

\begin{abstract}
Flight control laws of modern aircraft are scheduled with respect to flight point parameters. The loss of the air data measurement system implies inevitably the loss of relevant scheduling information. A strategy to design a fault tolerant longitudinal flight control system is proposed which can accommodate the total loss of the angle of attack and the calibrated airspeed measurements. In this scenario the described robust longitudinal control law is employed ensuring a control performance as close as possible to the nominal one. The controller is designed using quantitative feedback theory applied to the linear approximation of the longitudinal aircraft dynamics and is tested on a nonlinear high fidelity simulation model of a civil aircraft.
\end{abstract}

\section{Introduction}

Longitudinal control laws of civil aircraft guarantee high performance operation of the aircraft over the full range of operating points in presence of various parametric and operational uncertainties. Typically, a gain scheduling based controller is employed, where the main scheduling parameters are provided by the air data measurement system, as for example calibrated air speed and Mach number. Additionally, longitudinal control laws include protections on the aircraft's pitch angle, angle of attack, vertical load factor and air speed, which may also depend on scheduling variables provided by the air data system.

One of the recent trends in designing flight control systems is trying to preserve the longitudinal nominal law performance in the presence of faults ${ }^{1}$ (see also Ref. 2 for an alternative approach for the aircraft's lateral dynamics). By avoiding the switch to a so-called alternate or even direct control law, which both provide a very limited functionality (e.g., only pitch damping), the goal is to keep the nominal control performance even in the case of failures, without increasing the pilot's workload. In this paper we address the fault tolerant controller design problematic in the case of air data sensor faults. In more detail, the loss of the calibrated airspeed and angle of attack measurements is considered. A design strategy to derive a backup controller covering the whole flight envelope and providing satisfactory closed loop performance and stability characteristics independent of air data measurements is proposed.

The closed loop aircraft system, including the natural, i.e. unaugmented, dynamics of the aircraft, its control system as well as actuators and sensors, is depicted in figure 1. It clearly illustrates the central position of the sensors in the control loop. Due to their significant role for the implementation of a high performance and robust longitudinal control law, a triplex sensor redundancy is nowadays a standard requirement to accommodate single sensor faults in each of the air data measurements, as presented in Ref. 3 . In the considered scenario of a total loss of the calibrated airspeed and the angle of attack sensors one possibility is - after an adequate detection and isolation of the faults - the switch to a backup control law being independent of both measurements.

The case of simultaneous failures of two or more sensors of one measurement requires advanced fault diagnosis techniques to isolate the faulty sensors. An individual monitoring of the angle of attack (AoA) sensors has been proposed in Ref. 4 using all available measurements, including the calibrated airspeed. Simultaneous AoA sensor faults can be isolated using low order linear parameter varying residual generation filters. In Ref. 3 the individual monitoring of the six sensors, three for the calibrated air speed and three for

*Research Associate: Daniel.0ssmann@dlr.de 


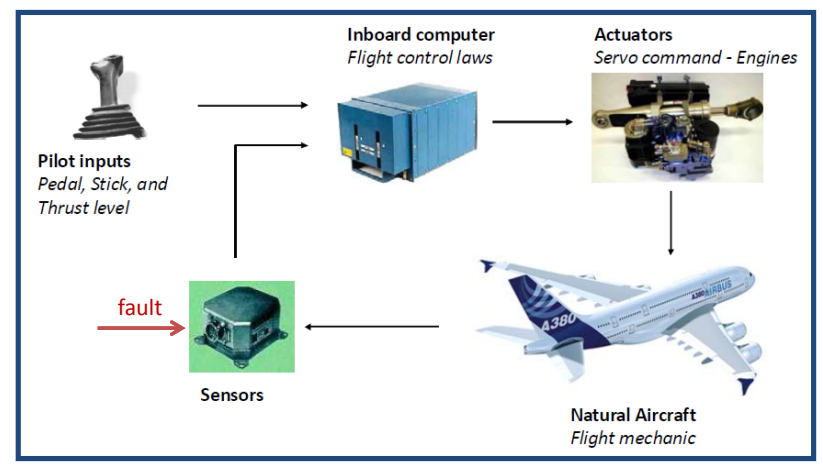

Figure 1. Closed loop aircraft system

the AoA, has been proposed. Robust fault isolation of all six potential sensor faults provides the possibility to use the healthy measurement(s) for the control and protection algorithms, as long as at least one sensor in each category is healthy. Further, the isolation sets the basis for the switching to a backup control law in case of a total failure.

The design of a robust backup controller in case of the total loss of the calibrated air speed and AoA measurements is a benchmark scenario formulated in the FP7 Project RECONFIGURE (Reconfiguration of Control in Flight for Integral Global Upset Recovery). ${ }^{5}$ In such a scenario maintaining a control law with the performance as close as possible to the nominal one and keeping the aircraft in a safe region appears to be a reasonable goal for the design of a backup control law. In this paper a robust backup longitudinal control law is designed using quantitative feedback theory to take uncertainties resulting from the loss of scheduling information into account. To fulfill the performance and stability requirements in the whole flight envelope a set of air data free scheduling variables is proposed.

\section{The reconfigurable fault tolerant longitudinal control architecture}

In this section we describe the proposed reconfigurable longitudinal flight controller as part of the fault tolerant control (FTC) architecture shown in figure 2 to manage the total loss of the air data measurements calibrated airspeed and angle of attack. A detailed description of the fault detection and diagnosis system, the supervisor and the reconfigurable signal consolidation can be found in Ref. 3. We will assume, that the fault detection and isolation as well as the switching information $\sigma_{c}$ is available and provided by the supervisor. The signal $\sigma_{s}$ in figure 2 is used in preliminary steps to reconfigure the sensor consolidation, which is based on the fault detection and isolation signal $\iota$. This procedure is not the topic of this paper and suitable fault detection and diagnosis systems for the given underlying aircraft dynamics are presented in Ref. 3. Any author interested in fault detection and diagnosis aspects and the reconfiguration of the sensor consolidation for the RECONFIGURE benchmark may refer to this publication and the publications cited therein. The relevant blocks and signals for control purposes depicted in figure 2 are descried in what follows.

\section{A. Open loop aircraft model}

The open loop aircraft model includes beside the aircraft dynamics suitable actuators to control the relevant control surfaces via the input signal $u$ and sensors providing the measurement signals $y$. The components of the control input vector $u$ are the demanded elevator and stabilizer deflections, while $y$ includes the main measurement signals as the calibrated airspeed and the angle of attack (both with triplex redundancy) as well as measurements used by the longitudinal control algorithm or the fault monitoring algorithms, as the longitudinal and vertical load factor, pitch rate, pitch angle, etc. The wind components $d$ and sensor faults $f$ are additional inputs which influence the aircraft behavior. Typical components of the flight parameter vector $\rho$ are the aircraft weight, the altitude, the mach number and center of gravity position. 


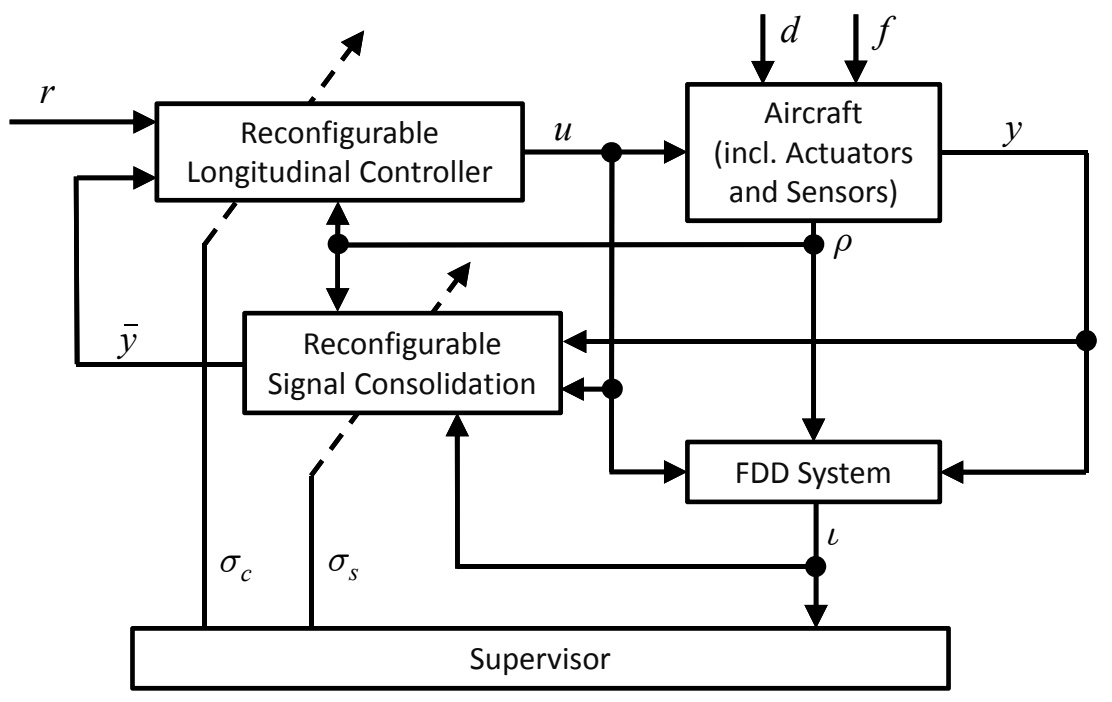

Figure 2. Fault detection and diagnosis based FTC architecture for the aircraft's longitudinal dynamics

\section{B. Reconfigurable longitudinal controller}

The reconfigurable longitudinal control system internally consists of two blocks, as shown in figure 3 . Both the nominal controller and the backup controller process the pilot reference signal $r$ and a set of consolidated measurements $\bar{y}$ to generate the actuator command $u$. While the nominal controller is usually gain scheduled with the scheduling parameters $\rho$, the backup controller will use a different set $\bar{\rho}$ of scheduling variables, which is independent of the aircraft's air data measurement system. The switching from the nominal to the backup controller is commanded via the switching signal $\sigma_{c}$.

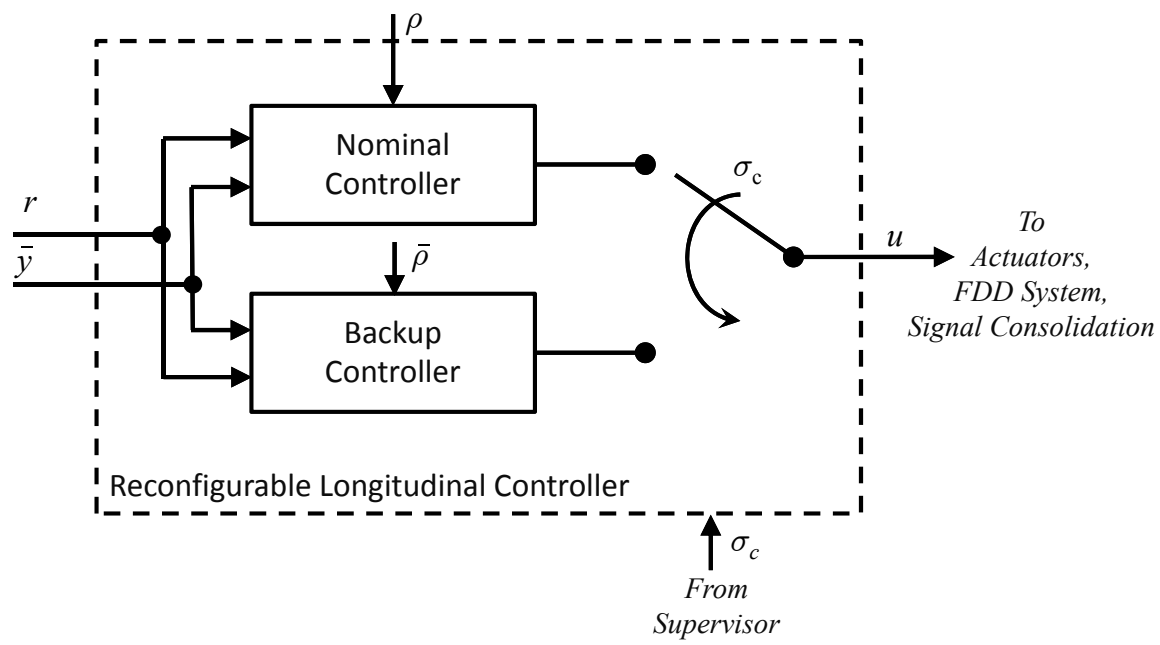

Figure 3. Reconfigurable longitudinal control system 


\section{Quantitative feedback theory}

Developed in the early 70s by Horowitz ${ }^{6}$ the quantitative feedback theory (QFT) is a robust control design technique which has been modified and improved in the last decades. It makes use of measurable plant outputs as feedback to generate a desired system response, considering quantified, structured plant uncertainties and disturbances.

The QFT method can be interpreted as a mixed open loop and closed loop shaping design method for uncertain systems. Compared to robust control design methods like the $\mu$-synthesis the methods is less complex but provides a deep insight in the controller design and structure throughout the whole design process. It is a method which provides an easy access to find the best controller for single-input singleoutput systems in a quite intuitive way. ${ }^{7}$ As other loop shaping methods the method tends to be an iterative procedure. The main drawback of the method is that these iterations may become intensive when it comes to multiple-input multiple-output (MIMO) systems. There has been a lot of effort and research to improve the design process for MIMO systems. However, in literature the claims of the theory are criticized to be unjustified for MIMO systems. ${ }^{7}$

The objective of this paper is not to discuss the legitimacy of the method but to demonstrate that in certain cases it can be easily applied to design a fault tolerant controller also in case of multiple feedback systems. Due to the multiple feedback loops attention has to be paid on the influence of each loop on the performance and stability characteristics to finally be able to design an adequate controller. In the following the basic concept of the QFT control design method and how it can be applied for the design of a fault tolerant controller is presented.

\section{A. Basics of the QFT design method}

The QFT method uses a unity feedback, a cascade compensator and a prefilter to reduce the variation of the plant output due to the defined parameter variations. The cascade controller is designed to ensure that the demanded stability and performance requirements are met, while the prefilter enables the possibility to meet the closed loop tracking requirements.

QFT is a frequency domain design technique in which tradeoffs between compensator complexity and performance can be visualized directly. Structured plant uncertainty is expressed in the Nichols chart on a set of fixed design frequencies by the so-called plant templates. ${ }^{6}$ The stability bounds are calculated using these templates together with the defined phase and gain margins. The tracking bounds are derived using the plant templates and upper and lower limits on the closed loop frequency response. The disturbance bounds are based on the plant templates and an upper limit only. The loop shaping is done based on the characteristics of the Nichols chart, as it offers the possibility to compare the open loop stability bounds as well as the closed loop performance bounds with the open loop dynamics only. Thus, a curve fitting problem in a two-dimensional plane needs to be solved by loop shaping.

The two degree of freedom feedback configuration including the feedback controller $C(s)$ and the prefilter $F(s)$ is depicted in figure 4. $\mathbf{r}(s), \mathbf{u}(s), \mathbf{d}(s)$ and $\mathbf{y}(s)$ are the Laplace transformed of the reference input signal $r(t)$, the plant input signal $u(t)$, the disturbance signal $d(t)$ and the system output signal $y(t)$. Note that all signals are scalars. In the following we assume that the parameter vector $p$ is constant. In that way we can define $G(s, p)$ as a parameter dependent transfer function. The plant uncertainty is described by the non measurable parameter vector $p \in \Pi$, where $\Pi$ is a set of parameter values. The closed loop transfer function from the reference input $\mathbf{r}(s)$ to the output $\mathbf{y}(s)$ is given by

$$
T(s, p)=G(s, p) C(s) F(s)(1+G(s, p) C(s))^{-1} .
$$

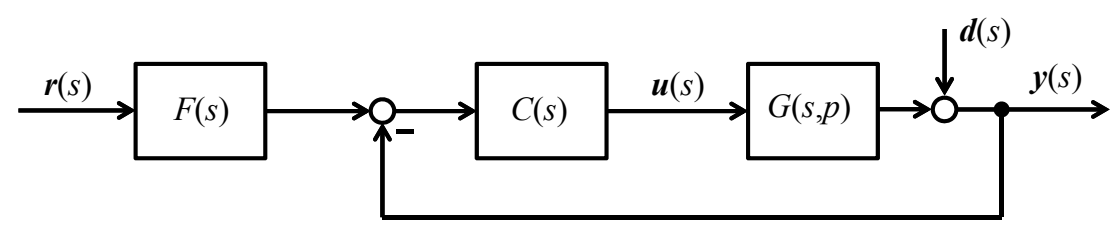

Figure 4. QFT controller structure 
In the frequency domain, the set of plants is given by

$$
\mathcal{G}_{\omega}=\{G(j \omega, p): p \in \Pi\} .
$$

For each frequency $\omega_{i}, \mathcal{G}_{\omega_{i}}$ defines a plant template, which describes the uncertainty of the plant at $\omega_{i}$ in terms of phase and magnitude. A set of $N$ frequencies $\left\{\omega_{1}, \omega_{2}, \ldots, \omega_{N}\right\}$, which has to cover the relevant frequency range of the plant, is chosen for the design of the controller. Having the plant templates available, the next step is to define the different controller design objectives, leading to the design bounds.

\section{Robust stability objectives}

One of the main objectives in robust control is that the controller delivers a stable closed loop system over the whole parameter space. In the Nichols chart, this basic stability requirement is fulfilled, if the open loop frequency responses $L(j \omega, p)=G(j \omega, p) C(j \omega)$ for al $p \in \Pi$ do not cross the critical point at $0 \mathrm{~dB}$ and -180 deg. In flight control, usually additional phase and gain margins to the critical point are requested to take unmodeled effects and uncertainties into account. These additional margins can be described by an M-circle - a circle of constant closed loop magnitude - in the Nichols chart. In this case the stability requirements are met if the open loop frequency responses $L(j \omega, p)$ for all $p \in \Pi$ do not penetrate the defined M-circle.

The idea of the QFT design is the following: instead of checking all possible open loop frequency responses against the defined M-circle at each frequency point, modified M-circles, the so-called $\mathcal{U}$-contours, are determined for a nominal open loop frequency response on the design frequency points $\omega_{i},{ }^{8}$ using the plant templates and the desired stability margins. If this nominal open loop frequency response lies outside the determined contours at the design frequency $\omega_{i}$, it is guaranteed, that all responses $L\left(j \omega_{i}, p\right)$ for $p \in \Pi$ do not penetrate the defined stability bounds at the frequency $\omega_{i}$. Instead of checking numerous open loop responses, only one nominal response has to be compared on different design frequencies with the dedicated contour plots. The aim is to design a controller, ensuring that at all design frequencies the nominal frequency responses lies outside the corresponding contour plot.

\section{Robust performance objectives}

Good closed loop performance can be quantified, among others, by good tracking of the reference signal and good disturbance rejection. The tracking objectives can be expressed by the set of inequalities

$$
\max _{p \in \Pi}\left|\frac{C\left(j \omega_{i}\right) G\left(p, j \omega_{i}\right)}{1+C\left(j \omega_{i}\right) G\left(p, j \omega_{i}\right)}\right|_{d B}-\min _{p \in \Pi}\left|\frac{C\left(j \omega_{i}\right) G\left(p, j \omega_{i}\right)}{1+C\left(j \omega_{i}\right) G\left(p, j \omega_{i}\right)}\right|_{d B} \leq \delta_{t}\left(\omega_{i}\right):=\left.b_{u}\left(\omega_{i}\right)\right|_{d B}-\left.b_{l}\left(\omega_{i}\right)\right|_{d B}
$$

for each $i=1, \ldots, N$, where $\delta_{t}\left(\omega_{i}\right)$ takes the difference of the maximum $\left(b_{u}(\omega)=\left|B_{u}(j \omega)\right|\right)$ and minimum $\left(b_{l}(\omega)=\left|B_{l}(j \omega)\right|\right)$ tracking boundary at the frequency $\omega_{i}$ into account. $B_{l}(j \omega)$ and $B_{u}(j \omega)$ denote the frequency responses describing the upper and lower tracking bounds. Note that only the difference between the maximum and minimum value of the gain response, hence the gain spread, is considered in (3) and thus used for the design of the controller $C(s)$. It is assumed, that any required absolute value of the gain can be achieved using the prefilter $F(s) .{ }^{9}$ In other words the controller $C(s)$ is used to ensure that the gain spread between the plants at the design frequencies is limited so that at all design frequencies the frequency responses can be placed within the defined tracking bounds using the prefilter $F(s)$.

The robust disturbance attenuation objective can be satisfied by bounding the sensitivity function

$$
\max _{p \in \Pi}\left|\frac{1}{1+C\left(j \omega_{i}\right) G\left(p, j \omega_{i}\right)}\right| \leq b_{d}\left(\omega_{i}\right)
$$

for each $i=1, \ldots, N$, where the bound on the disturbance to the output transfer is usually defined by the magnitude of a given frequency response $b_{d}(\omega)=\left|B_{d}(j \omega)\right|$.

The robust performance inequality constraints (3) and (4) regarding the closed loop gain are transferred into constraints of the uncertain open loop response $L(j \omega, p)$, resulting in a number of contour plots for each design frequency $\omega_{i}$. These contours are the so-called Horowitz-templates $t_{i}^{t}(\phi)$ for tracking and the disturbance rejection templates $t_{i}^{d}(\phi)$ for $i=1, \ldots, N$, where $\phi$ describes the range of the open loop phase in the Nichols chart. Usually the boundaries are calculated for the discrete set of $N_{\phi}$ open loop phase points $\phi_{i} \in \Phi$, where the range is limited to $\Phi=[-360,0] \mathrm{deg}$. The gain spread is robustly limited on a design point is achieved if the nominal open loop frequency response fulfills $\left|L\left(j \omega_{i}\right)\right|_{d B} \geq t_{i}^{t}\left(\phi_{i}\right)$, where 
$\arg \left(L\left(j \omega_{i}\right)\right)=\phi_{i}$. Robust disturbance rejection is fulfilled if $\left|L\left(j \omega_{i}\right)\right|_{d B} \geq t_{i}^{d}\left(\phi_{i}\right){ }^{9}$ If the conditions are fulfilled for all frequencies $\omega_{i}$, the gain spread for the robust tracking performance and the disturbance rejection and the design frequencies are adequately chosen, one can suppose, that the requirements are fulfilled on each point of the relevant frequency range. However, for validation purposes an assessment of the controller has to be done. Note that further design objectives are available as for example to limit the high frequency sensors noise influence or the required control magnitude of the actuators. ${ }^{8}$

\section{B. Quantitative feedback theory for fault tolerant control}

The QFT has been developed to deal with plant uncertainties resulting from modeling errors, neglected dynamics or nonlinear effects. In this paper we formulate the fault tolerant control problem as design problem which can be solved by QFT: the loss of scheduling information unavoidably results in an uncertain plant for which a robust controller has to be designed. In other words, the designed controller has to be robust for a set of flight conditions, for which the design of a gain scheduling controller is not possible any more due to the lack of scheduling information. In this way the uncertainties are a direct result of changing the flight condition. To provide a simple controller but still guarantee robust stability and adequate flying quality performance in the whole flight envelope, a subset from the remaining available flight parameter measurement variables is selected as scheduling variable and used in a multi-model controller design.

\section{Fault tolerant controller synthesis}

\section{A. Synthesis models}

A set of $N=214$ linearized models of the form

$$
\begin{aligned}
& \dot{x}^{(i)}(t)=A^{(i)} x^{(i)}(t)+B_{u}^{(i)} u(t)+B_{d}^{(i)} d(t) \\
& y^{(i)}(t)=C^{(i)} x^{(i)}(t)+D_{u}^{(i)} u(t)+D_{d}^{(i)} d(t)
\end{aligned}
$$

of the open loop aircraft dynamics in the dedicated flight envelope is available. In $(5) x^{(i)}(t)$ and $y^{(i)}(t)$ are, respectively, the state and the output vector of the $i$-th system, and $u(t)$ and $d(t)$ are the control and disturbance input vectors.

The models of the aircraft have been generated on different trim points which are defined by nominal values of the aircraft weight, center of gravity position, velocity and the altitude. All aircraft models are of 5 th order with the states angle of attack, pitch rate, ground speed, pitch angle and aircraft altitude. Among others, the output vector $y$ includes the five states, the vertical velocity and the accelerations in the $x$ and $z$ direction of the aircraft. The elements of the input vector $u$ are the stabilizer position, the four elevator deflections and the thrust input. As measurable parameters the weight and the center of gravity of the aircraft are available. Linear, second order models of actuators, first order models of sensors and higher order structural filters are available, leading to a high order linear open loop system. The structural filters are implemented due to the size of the aircraft to filter out any unwanted oscillations in the measurement signals which could excite the aircraft's eigenmodes. The nominal controller is a kind of $C^{*}$ (spoken: C-star) controller, which uses in contrast to a classical $C^{*}$ control law a pure load factor command signal. This controller will be used as benchmark for the definition of the design bounds and later for the validation of the designed backup controller in the nonlinear simulation model.

The selection of the new scheduling variables is based on flying and handling quality criteria. These criteria were originally developed for the natural aircraft's short period motion, which can be expressed by the second order model

$$
\begin{aligned}
& {\left[\begin{array}{c}
\dot{\alpha} \\
\dot{q}
\end{array}\right]=\left[\begin{array}{cc}
Z_{\alpha} & Z_{q} \\
M_{\alpha} & M_{q}
\end{array}\right]\left[\begin{array}{l}
\alpha \\
q
\end{array}\right]+\left[\begin{array}{c}
Z_{\eta} \\
M_{\eta}
\end{array}\right] \eta} \\
& {\left[\begin{array}{c}
\alpha \\
q \\
n_{z}
\end{array}\right]=\left[\begin{array}{cc}
1 & 0 \\
0 & 1 \\
-\frac{V}{g} Z_{\alpha} & 0
\end{array}\right]\left[\begin{array}{l}
\alpha \\
q
\end{array}\right]+\left[\begin{array}{c}
0 \\
0 \\
-\frac{V}{g} Z_{\eta}
\end{array}\right] \eta .}
\end{aligned}
$$

In (6) $Z_{\alpha}, Z_{q}$ and $Z_{\eta}$ resp. $M_{\alpha}, M_{q}$ and $M_{\eta}$ are the force and moment coefficients due to a change in the angle of attack $\alpha$, pitch rate $q$ and elevator deflection $\eta$, respectively. $V$ represents the aircraft's true 
air speed, $g$ is the gravitational constant and $n_{z}$ is the vertical load factor. The low order form (6) has to be used to be able to validate an aircraft configuration using classical flying and handling quality criteria. However, modern aircraft systems are higher order systems due to additional dynamics of actuators, sensors and additional pre- and shaping-filters as well as the controller. The so-called equivalent system concept, stated generally, is simply to match the higher order dynamics with a low order equivalent. For conventional aircraft response types the equivalent system has the same form as the natural aircraft plus a time delay to approximate the higher order phase lags of the system components. ${ }^{10}$
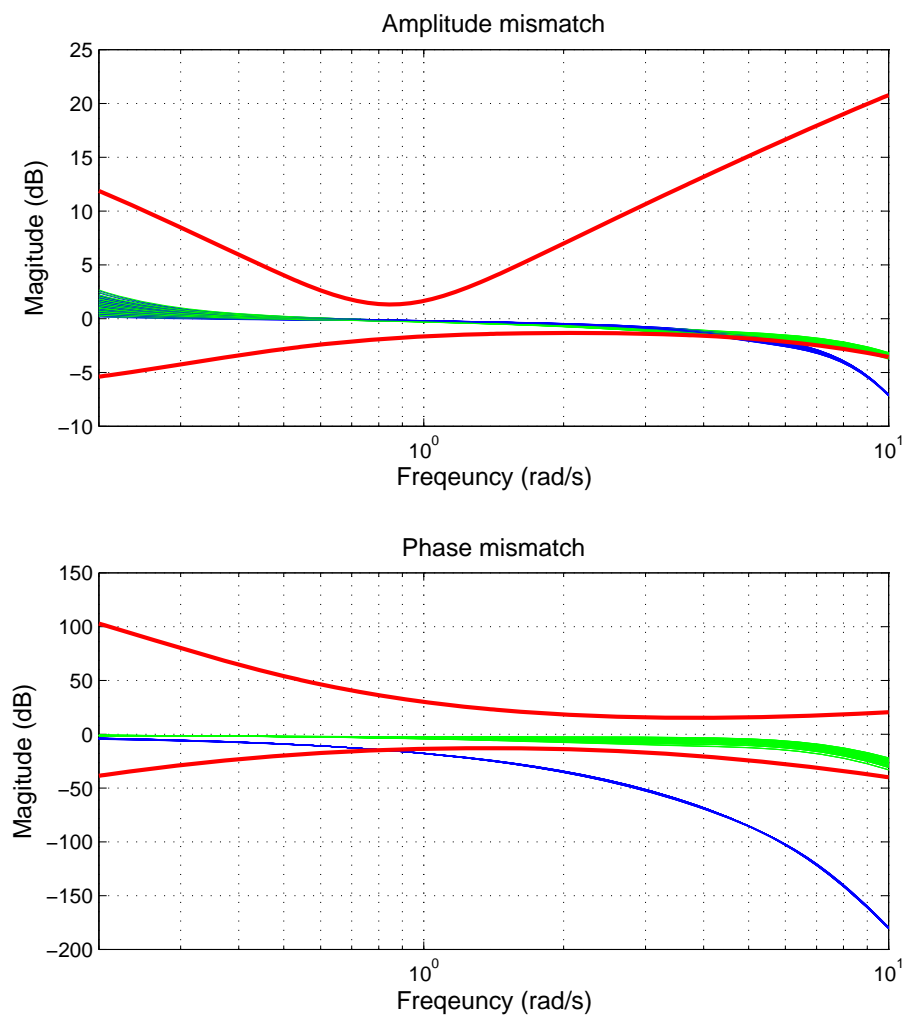

Figure 5. Low order system validation in the Bode plot

In figure 5 the allowed boundaries for the difference (or mismatch) between the higher order system and the lower order system in magnitude and phase are depicted in red. In case these difference lies between the bounds the pilot does not feel any difference between the two systems. The difference between the full order models including sensors, actuators and filters and the second order models of the longitudinal motion (6) directly derived from the 5th order model (i.e. without adapting the entries of the state space matrices of the low order approximation) of the aircraft dynamics for 44 responses belonging to a lower level flight condition are depicted in figure 5 in blue. It is clearly visible that at higher frequencies especially the approximation of the phase response is not good enough. For a flying and especially handling quality evaluation of the aircraft using traditional criteria, there would be a need to determine a better low order equivalent system (LOES). However, the additional phase lag mainly changes the time delay in the LOES and has little influence on the short period damping and the eigenfrequency. ${ }^{11}$ As the control anticipation parameter, the short period damping and the value of $n_{z \alpha}$ are not influenced by the time delay, the use of the second order longitudinal model for the selection of the scheduling variables based on the mentioned flying quality parameters is sufficient.

The green lines correspond to the difference between the full order models and short period dynamics plus all sensor, filter and actuator dynamics, only neglecting the phygoid dynamics. This difference lies in the defined bounds, as the phygoid only influences the dynamics at very low frequencies. Hence, this reduced 
models can be used for the controller design applying the QFT method as well as for the linear analysis of the closed loop, which makes the determination of the overshoots and settling time of the short period motion straightforward.

\section{B. QFT design requirements}

The next step in the design process is the definition of the stability and performance bounds. Therefore the controller structure is discussed first. As the aircraft augmented with the backup controller should show characteristics as close as possible to the nominal controller, the use of a $C^{*}$ based control law becomes obvious. The $C^{*}$ signal is a mixture of the vertical load factor $n_{z}$ and the pitch rate $q$

$$
C^{*}(t)=\frac{V_{m}}{g} q(t)+n_{z}(t),
$$

where $V_{m}$ is the average of the operating velocity range. Instead of a pure $C^{*}$ feedback controller we will add a pitch damper in the inner loop to ensure a minimum damping ratio. This features a kind of a direct link control with a pitch damper in case also the backup controller has to be switched off, what might be necessary if the $n_{z}$ measurement is lost.

As mentioned at the beginning of section IV the nominal control law is not a classical $C^{*}$ control law as it features a pure load factor reference system. The mixture of the load factor and the pitch rate as described in (7) is only used in an internal feedback loop. However, the backup controller will use a classical $C^{*}$ control law with $C^{*}$ as reference signal. The required robust stabilization for different aircraft velocities together with the compliance with the performance specifications could not be achieved using a pure load factor command system.

\section{Robust stability objectives}

The stability specifications for the backup controller design defined in the RECONFIGURE project request a phase margin of $60 \mathrm{deg}$. The phase margin is defined as distance of the frequency response to the critical point $(0 \mathrm{~dB} /-180 \mathrm{deg})$ at an open loop gain of $0 \mathrm{~dB}$, hence $\angle\left(L\left(j \omega_{c}\right)\right)+180$, where $\omega_{c}$ is the so-called cross over frequency describing the frequency where $|L(j \omega)|$ crosses $0 \mathrm{~dB}$ for the first time from above in the Bode plot or Nichols chart. The requested gain margin is $6 \mathrm{~dB}$. The gain margin is defined as distance of the frequency response to the critical point at $-180 \mathrm{deg}$, hence $-\left|L\left(j \omega_{180}\right)\right|_{d B}$, where $\omega_{180}$ is the so-called phase cross over frequency, ${ }^{12}$ describing the frequency where the open loop frequency response $L(j \omega)$ crosses the negative real axis between -1 and 0 in the Nyquist plot. ${ }^{12}$ Note that these requirements are defined at two discrete points of the frequency response, namely at $|L(j \omega)|_{d B}=0$ and $L L(j \omega)=-180 \mathrm{deg}$. This differs from the QFT design criteria where the phase and magnitude margins are requested at each frequency design point, resulting in the so-called $\mathcal{U}$-contours, which are similar to the classical Nichols diamond used for flight control development for a single LTI system. Hence, using the QFT design method and its bounds the design tends to be more conservative.

\section{Robust performance bounds}

The performance specifications are expressed by characteristics of the closed loop step responses. The requirements are a maximum pitch rate overshoot of $30 \%$, a maximum load factor overshoot of $10 \%$, a maximum load factor settling time of the $6 \mathrm{~s}$ and a uniform control anticipation parameter in the whole flight envelope. The conversion of these time domain criteria into the frequency domain criteria is not a trivial task. Although there exist some $C^{*}$ bounds in literature, they were developed for military aircraft. While these bounds where successfully applied for the control design for the Boeing 747 aircraft in Ref. 13 it was also shown that the design of the controller has to be close to the lower $C^{*}$ bound (and thereby limit the bandwidth of the aircraft) to achieve satisfactory flying qualities.

As the aircraft dealt with in this paper is even larger it may require a stricter bandwidth constraint. Because of these aspects and the fact, that the closed loop behavior using the backup controller should be as close as possible to the behavior of the nominal controller, the performance bounds are computed using the linear aircraft model augmented with the nominal controller. This is further supported by the fact, that the design constraints for the nominal controller were equal to the ones used for the backup controller. The range of resulting $C^{*}$ frequency responses define the performance bounds for the QFT design. The results 
of the boundary determination are depicted in figure 6 . The blue lines denote all $214 C^{*}$ magnitude plots, while the red lines define the minimum and maximum magnitude value at each frequency. The green lines are the approximated bounds for the QFT controller design. Around the bandwidth frequency (defined at the point where the closed loop frequency response crosses the $-3 \mathrm{~dB}$ from above) the frequency responses shall fall with $-20 \mathrm{~dB} /$ decade to ensure a fast rolloff and good noise attenuation at higher frequencies. ${ }^{12}$ This results in a target bandwidth between 0.4 and $2 \mathrm{rad} / \mathrm{s}$ for all models. As expected, these bounds lie below the classical $C^{*}$ bounds, which were developed for high maneuverable aircraft.

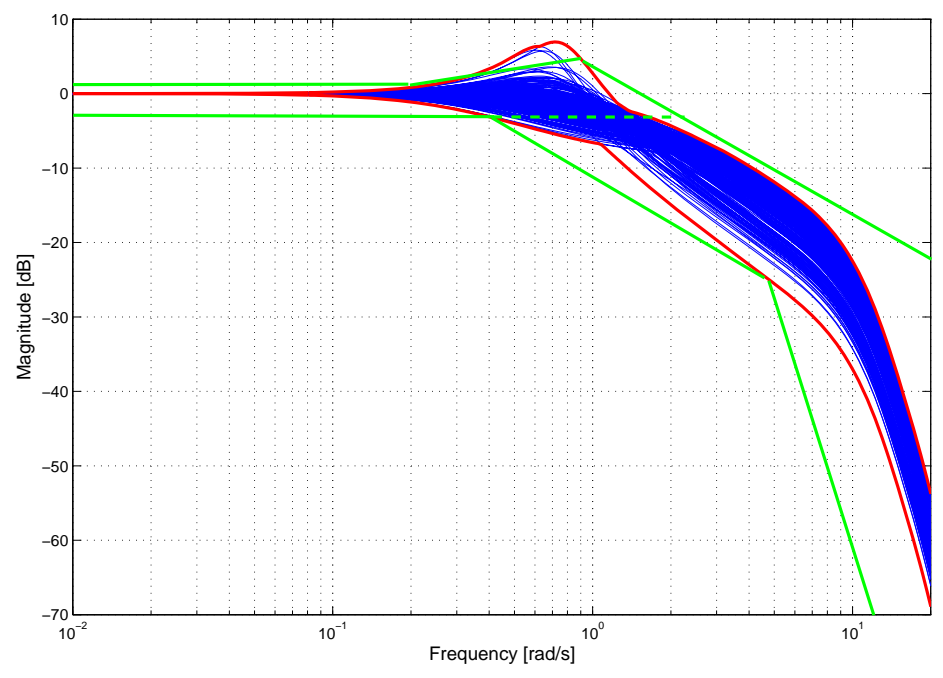

Figure 6. Approximated tracking bounds ed on the nominal closed loop responses

It can be assumed, that the compliance with the presented requirements, which are based on the specifications of the nominal controller, provides a satisfactory closed loop system. Due to this fact the disturbance bounds as well as the bounds to limit the control input amplitude are neglected in the description of the design process in this paper. In fact, the bounds were included in the design process but the stability and tracking requirements emerged the critical ones to be met.

\section{Selection of scheduling parameters}

A basic flying quality validation of the aircraft's open loop using the second order approximation (6) of the short period dynamics serves as basis for the selection of the scheduling parameters. Classical flying quality parameters defined in the MIL-specifications ${ }^{14}$ are the short period damping $\zeta$, the short period frequency $\omega_{0}$ and the value of $n_{z, \alpha}$ defined by

$$
n_{z \alpha}=\frac{n_{z}(\infty)}{\alpha(\infty)},
$$

describing the stationary load factor response by an angle of attack change of 1deg. Note that this value cannot be changed by any feedback loop on the aircrafts elevator like the one used in this paper. ${ }^{10}$ This fact will play a significant role when selecting the scheduling variables. The fourth parameter is the so-called control anticipation parameter (CAP) which is defined by

$$
C A P=\frac{\dot{q}(0)}{n_{z}(\infty)} \approx \frac{\omega_{0}}{n_{z \alpha}},
$$

describing how good the pilot is able to control the short period motion. ${ }^{10}$ The evaluation results of these four criteria for the 214 models of the natural second order aircraft dynamics are depicted in the two diagrams in figure 7. The left diagram is the classical MIL specification diagram for the short period frequency requirement for flight phase category $\mathrm{B}$, referring to non-terminal flight phases like cruise. The results indicate a Level 1 or Level 2 behavior for most of the natural aircraft dynamics. The three different colors 
refer to the three different center of gravity positions, starting from the minimum value in magenta to the maximum value in green. Note that the blue lines in the diagram indicate a constant CAP value. Hence, for a given center of gravity position the CAP value of the natural aircraft is quite uniform. To achieve a uniform CAP over the whole envelope different controllers are required for different center of gravity positions. This is confirmed by the right diagram, which shows the requirements on the damping ratio over the CAP.

One problematic region for a robust control design can be encountered for large center of gravity positions, depicted in green. There exists a large spread in the CAP and $\omega_{0}$ values, induced by low CAP combining with low $n_{z \alpha}$ values. As $n_{z \alpha}$ cannot be changed by the feedback on the elevator of the aircraft, this leads to a rather difficult control problem. To improve the situation, a scheduling variable to separate high and low $n_{z \alpha}$ values is needed. This separation is provided using the mass of the aircraft as depicted in the two diagrams in figure 8 , where low mass values are plotted in blue and high mass values are plotted in green. Although the mass does not provide a complete separation of the lower and higher $n_{z \alpha}$ values it can be used to separate the more difficult dynamics to control from the remaining ones. This approach ensures that it is not necessary to degrade all of the dynamics, showing already good flying qualities, due to some bad points in the flight envelope. Separating the center of gravity in 3 regions and the mass in 2 regions results in only 5 regions for the controller design, as low center of gravity positions do not combine with high mass values.
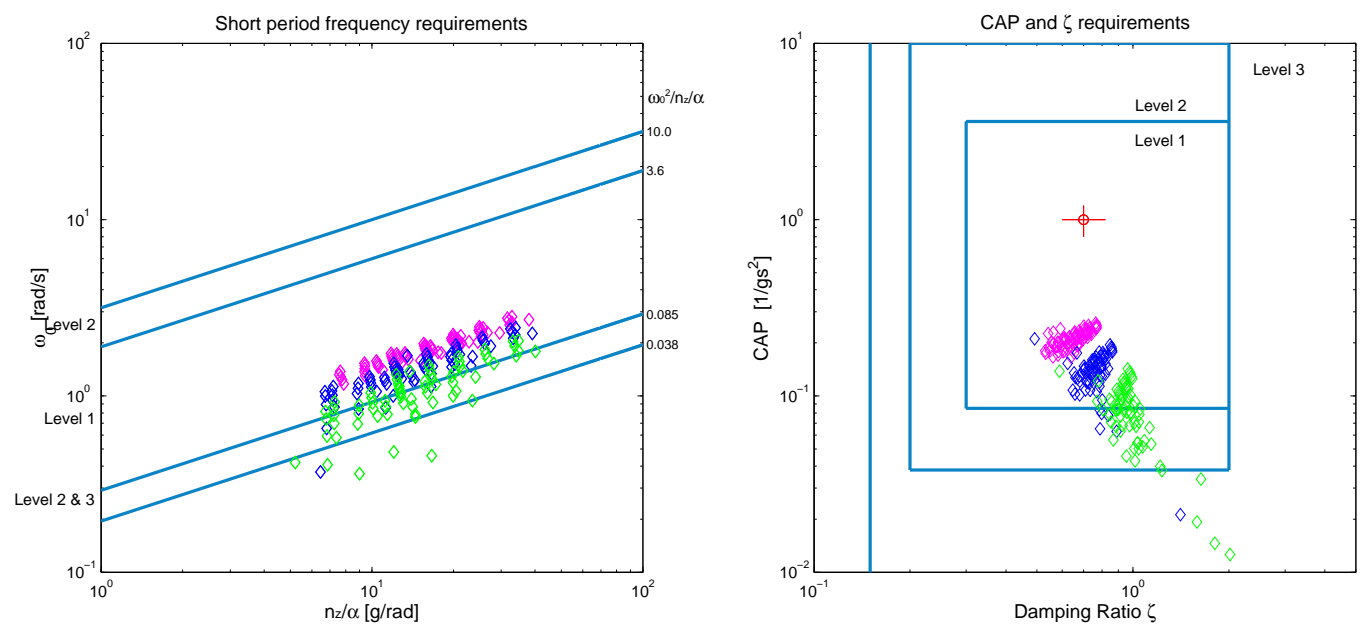

Figure 7. Dependency of $n_{z \alpha}$, eigenfrequency, damping and CAP value on center of gravity
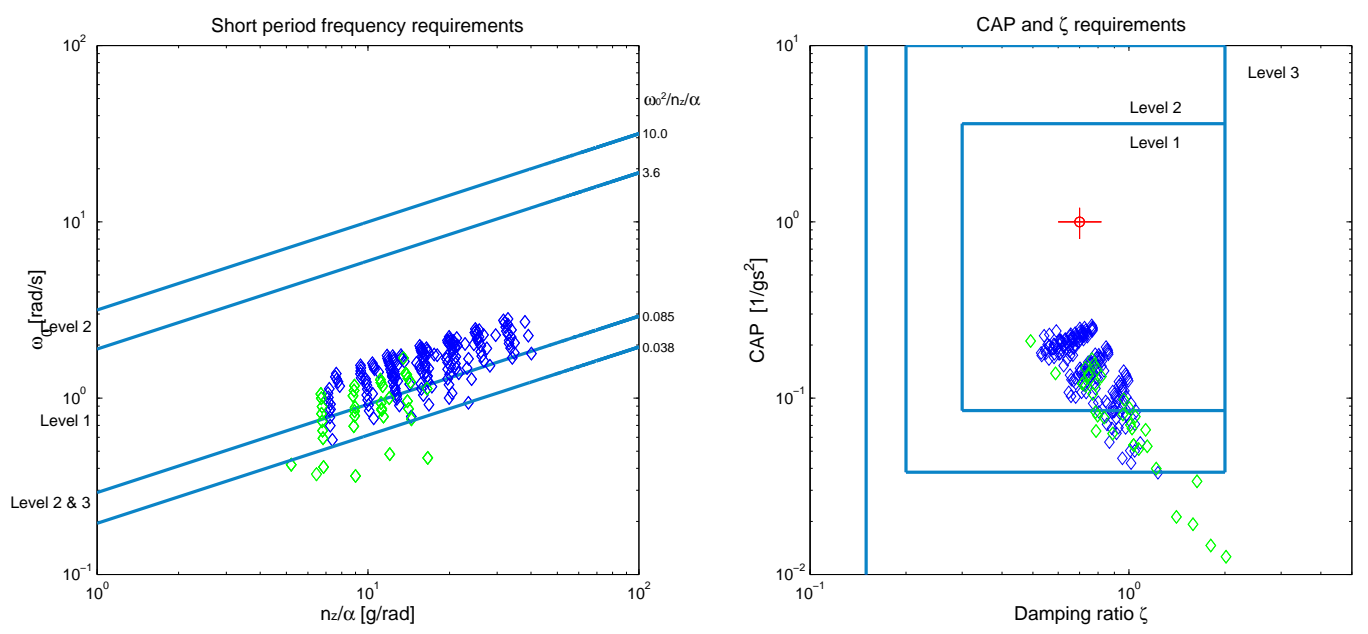

Figure 8. Dependency of $n_{z \alpha}$, eigenfrequency, damping and CAP value on mass 


\section{Feedback controller design}

The feedback controller of the FTC system consists of two feedback loops: to ensure a minimum damping ratio a constant pitch damper $K_{q}$ is implemented first. The second feedback loop features the $C^{*}$ feedback. Following the QFT design procedure, the two loops are designed one after each other. The pitch damper will be constant for all flight conditions. This basic pitch damper has several advantages: in case of losing the load factor measurement it can be used to damp the longitudinal motion in a direct link mode. Further, some of 214 aircraft dynamics show an unstable open loop behavior, so the pitch damper is used to robustly stabilize the aircraft at all flight conditions. Beside the positive effect of a robustly stabilized aircraft, this makes the design of the second feedback loop easier. ${ }^{15}$ Of course, this plus does not come without any issues the designer has to keep in mind: With the QFT methodology the controller is designed for the defined open loop plant. During the design of the second feedback loop this open loop plant will already have a pitch damper considered. Hence, the stability margins set for the open loop plant during the second design step will be different from the real ones. These real margins are determined using the loop which is cut open after the whole control system at the actuator position.

Therefore we will examine the differences of the two open loops first. In figure 9 the block diagram used for the stability analysis is depicted. In figure $9 \mathbf{q}(s), \mathbf{C}^{*}(s), \eta(s)$ and $\mathbf{q}_{\mathbf{c}}(s)$ are the Laplace transformed of the pitch rate signal $q(t)$, the $C^{*}$ signal $C^{*}(t)$, the elevator position signal $\eta(t)$ and the output signal $q_{c}(t)$ of $K_{C^{*}}$. For stability analysis, the required open loop at the actuator position can be expressed by

$$
L_{\eta}(s)=K_{q}\left(G_{q \eta}(s)+K_{C^{*}}(s) G_{C^{*} \eta}(s)\right),
$$

where $G_{q \eta}(s)$ and $G_{C^{*} \eta}(s)$ are the transfer functions from the elevator input $\eta(s)$ to the pitch rate $\mathbf{q}(s)$ and $\mathbf{C}^{*}(s)$, respectively, and $K_{C^{*}}(s)$ denotes the control element of the second feedback loop.

However, in the QFT design procedure the loop is always cut directly after the controller which is designed. In the design of the $C^{*}$ feedback loop the plant is already augmented with the pitch rate feedback gain (see figure 9). Hence the loop is cut after $K_{C^{*}}(s)$ at $\mathbf{c}(s)$. The open loop transfer function at $\mathbf{q}_{\mathbf{c}}(s)$ for the QFT design is given by

$$
L_{q_{c}}(s)=-K_{C^{*}}(s) G_{C^{*} q_{c}}(s),
$$

where $G_{C^{*} q_{c}}(s)$ is the transfer function from $\mathbf{q}_{\mathbf{c}}(s)$ to $\mathbf{C}^{*}(s)$, thus augmented with $K_{q}$.

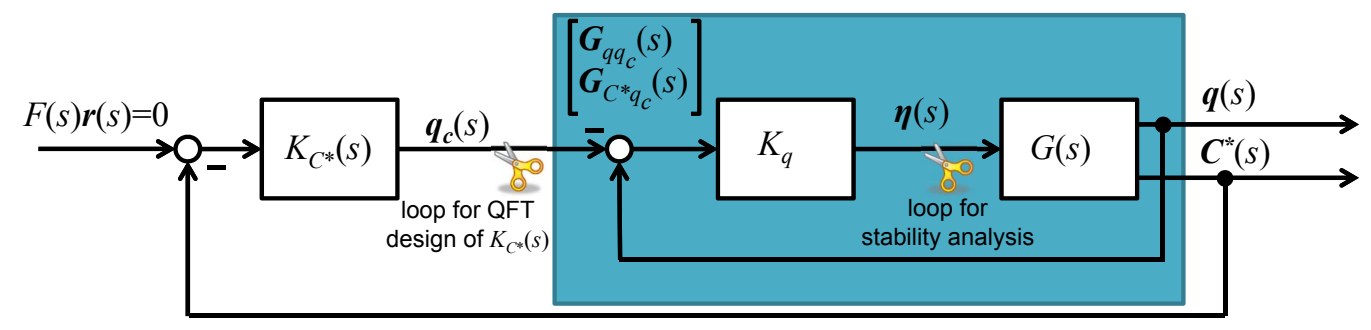

Figure 9. Position of loop cuts for the QFT design (left) and stability margin analysis (right)

As obviously (10) and (11) have different frequency responses, fulfilling the design margins during the design of the second controller $K_{C^{*}}(s)$ based on the open loop (11) does not mean that the frequency response of (10) fulfills them as well. However one can tell what happens by looking at both transfer functions: In equation (11) the augmented plant $G_{C^{*} q_{c}}(s)$ shows a different but similar pole location than $G_{C^{*} \eta}(s)$ in (10). Mainly the poles of the short period mode are modified, but as the gain $K_{q}$ tends to be small they are quite similar. The situation is different when looking at the zeros: The zeros of the two equations will be different, as in (10) their location is influenced by the numerator of $G_{q \eta}(s)$. Assuming a first order integral in the denominator of $K_{C^{*}}(s)$, which is common for PI or PID controller usually used in flight control, the influence of $s\left(s T_{\theta}+1\right)$, the numerator of $G_{q \eta}(s)$ multiplied with $s$, the denominator of $K_{C^{*}}(s)$, leads (in standard aircraft configurations) to a shift of the zeros of $G_{C^{*} \eta}$ towards the origin. This results in a reduced phase loss at low frequencies (i.e. higher stability margins) and a slightly increased amplitude at high frequencies (i.e. lower amplitude margins) of the transfer function $L_{\eta}(s)$ compared to $L_{q_{c}}(s)$. Hence, the use of the open 
loop transfer function $L_{q_{c}}(s)$ for the QFT design process may introduce some conservativeness regarding the phase, while the negative influence on the gain margin can be neglected.

Keeping this in mind, the 5 controllers for the different flight envelope regions are designed. In the following the design process for the first flight region at low mass and the minimum center of gravity position is presented. It turned out that for all flight regions the same controller structure with a different parameter setting can be used. In the linear and nonlinear assessment the results of all 5 flight regions will be discussed.

The first loop is designed as a constant gain $K_{q}=0.8$ for the whole flight envelope, ensuring a basic damping of the short period mode. The design of the second loop resulted in the structure of

$$
K_{C^{*}}(s)=K_{c} \frac{T_{z, c} s+1}{s},
$$

which is a classical proportional integral controller (PI), with the controller gain $K_{c}$ and the time constant $T_{z, c}$. In figure 10 the resulting contour plots based on the design requirements together with the response of nominal open loop plant augmented with the controller are depicted. By choosing an adequate parameter setting of the controller (12) an open loop response is achieved, which does not violate these contours. While at lower frequencies (e.g., below the desired system bandwidth) the performance as well as the stability bounds are relevant, at higher frequency the stability bounds are the main factor the designer needs to focus on.

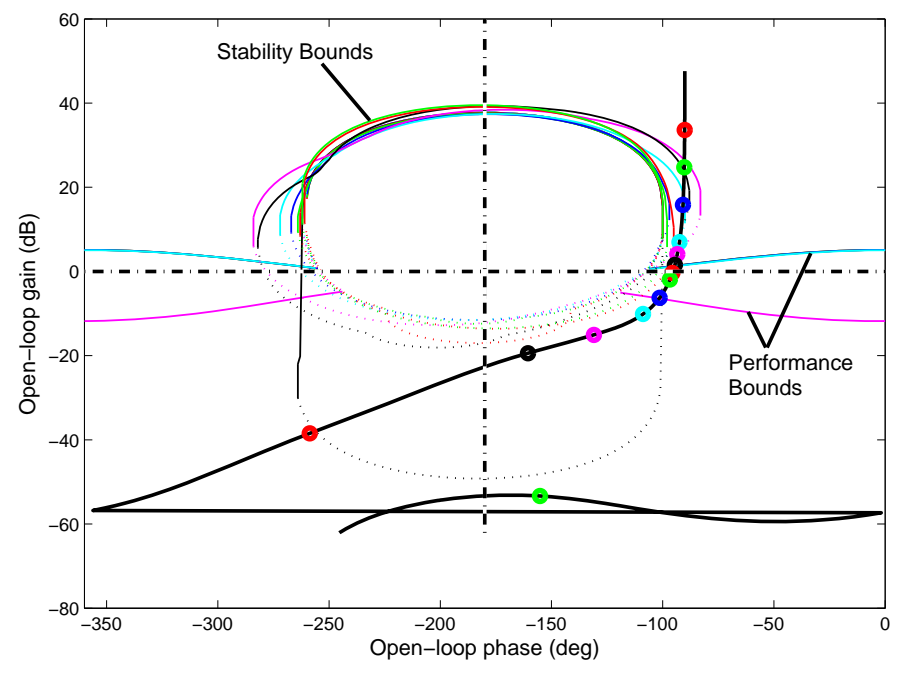

Figure 10. Fitted nominal plan with tracking and stability contours in the Nichols plot

\section{E. Prefilter design}

The designed feedback loops ensure the fulfillment of the stability requirements and limits the maximum gain spread relevant for the tracking bounds. The prefilter is used to bring the absolute gain values of the closed loop inside the tracking bounds. The same prefilter structure can be used in the different regions of the flight envelope with different settings of the parameter values. The prefilter resulted in a form of

$$
F(s)=\frac{T_{z, f} s+1}{\left(T_{p 1} s+1\right)\left(T_{p 2} s+1\right)} .
$$

In figure 11 the resulting upper and lower limit of all closed loop responses of the augmented plant in the first envelope region is depicted in blue together with the absolute bounds for the tracking performance in green. The frequency responses lie within the bounds indicating a satisfactory tracking behavior. 


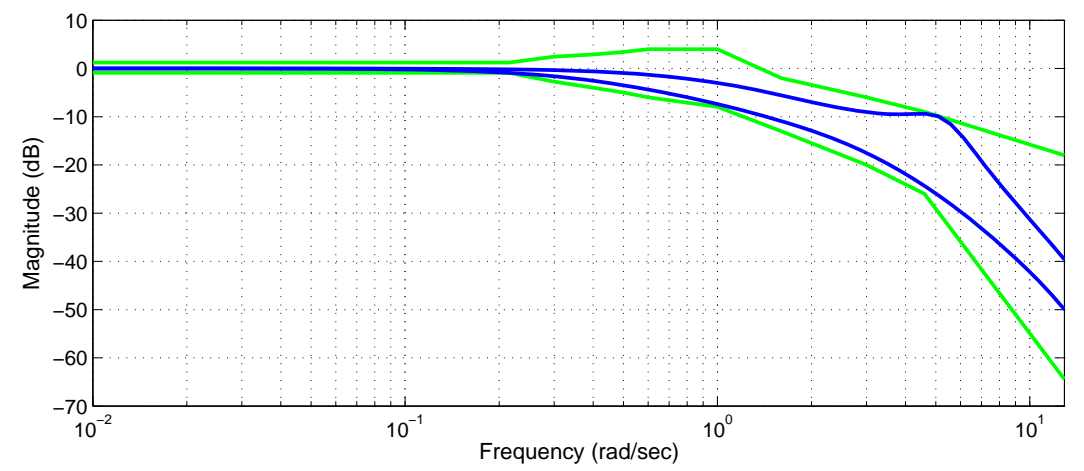

Figure 11. Tracking boundaries (green) and upper and lower limit of the fitted frequency responses (blue)

\section{F. Analysis of the resulting controller structure}

The elevator deflection $\eta(s)$ depending on $\mathbf{r}(s)$, the Laplace transformed reference signal $r(t)$, as well as on the outputs $\mathbf{C}^{*}(s)$ and $\mathbf{q}(s)$ is given by

$$
\eta(s)=K_{q}\left(\mathbf{q}(s)+K_{c} \frac{T_{z, c} s+1}{s}\left(F(s) \mathbf{r}(s)-\mathbf{C}^{*}(s)\right)\right),
$$

with the reference input $\mathbf{r}(s)=\mathbf{C}_{\mathbf{r}}^{*}(s)$, where $\mathbf{C}_{\mathbf{r}}^{*}(s)$ is the Laplace transformed of the reference signal $C_{r}^{*}(t)$. Rewriting this equation leads to

$$
\eta(s)=K_{q} \mathbf{q}(s)+K_{C^{*}} \mathbf{C}^{*}(s)-H_{C^{*}} F(s) \mathbf{C}_{\mathbf{r}}^{*}(s)-\frac{K_{i}}{s}\left(F(s) \mathbf{C}_{\mathbf{r}}^{*}(s)-\mathbf{C}^{*}(s)\right),
$$

where the feedforward gain is given by $H_{C^{*}}=K_{c} T_{z, c}$ and is equal to the feedback gain $K_{C^{*}}=H_{C^{*}}$. The gain of the integral action is given by $K_{i}=K_{q} K_{c}$. Equation (15) represents a feedback of the $C^{*}$ signal and the pitch rate with integral action on the reference error $\mathbf{e}_{r}(s)=F(s) \mathbf{C}_{\mathbf{r}}^{*}(s)-\mathbf{C}^{*}(s)$ as well as a feedforward gain to speed up the response. The form (15) indicates that the resulting controller has the same controller structure as the classical $C^{*}$ control law structure presented for example in Ref. 16.

\section{Controller assessment}

\section{A. Linear Analysis}

The linear analysis includes the linear simulation of the second order model of the aircraft dynamics plus sensor, actuator and filter dynamics as well as the determination of the stability margins using the corresponding open loop (10). As the phygoid mode mainly influences the low frequency part the influence on the stability margins is neglected. This enables to adequately determine overshoot and settling time values of the short period dynamics, which is not straight forward when including the phygoid motion.

For the validation of the performance in the time domain the load factor overshoots, the pitch rate overshoots, the CAPs and the load factor settling times are calculated. To validate the robustness regarding stability the gain and phase stability margins are computed. In table 1 the worst case results provided by the linear analysis for each flight envelope region is listed. Recall the request of a 60deg phase margin and a $6 \mathrm{~dB}$ gain margin. The minimum values over all points at each design point indicate that the robustness criteria are fulfilled. For the performance values, that is the overshoot in pitch rate and load factor as well as the load factor settling time with their required values of $30 \%, 10 \%$ and 6 seconds, the worst case (i.e. maximum) values are listed. In general the requirements are fulfilled and the small violations result from single flight points at the edge of the flight envelope. For the CAP a uniform behavior for the whole flight envelope is achieved as the minimum value over all point is 0.030 and the maximum is 0.067 . The last two design regions correspond to the maximum center of gravity position with CAP values between 0.03 and 
0.048. Note that the resulting CAP values lie in the Level 2 region of the flying quality criterion, although the behavior is quite similar to the nominal controller. This indicates that the bounds regarding the flying quality levels need to be adapted for modern aircraft with very high weights.

\begin{tabular}{|l|c|c|c|c|c|c|}
\hline & \multicolumn{2}{|c|}{ Margins } & \multicolumn{2}{c|}{ Overshoots } & $n_{z}$-Settling time & CAP \{min, max $\}$ \\
& Phase $(\mathrm{deg})$ & Gain $(\mathrm{dB})$ & $q(\%)$ & $n_{z}(\%)$ & $(\mathrm{sec})$ & $(\mathrm{gsec})^{-1}$ \\
\hline Envelope region 1 & 71 & 7.3 & 13.5 & 0 & 6.1 & $\{0.047,0.067\}$ \\
Envelope region 2 & 61 & 7.3 & 30 & 4 & 5.5 & $\{0.044,0.071\}$ \\
Envelope region 3 & 60 & 9.7 & 28 & 2 & 6.6 & $\{0.043,0.057\}$ \\
Envelope region 4 & 60 & 9.3 & 32 & 10 & 6.2 & $\{0.030,0.048\}$ \\
Envelope region 5 & 60 & 8.6 & 29 & 8 & 7 & $\{0.030,0.040\}$ \\
\hline
\end{tabular}

Table 1. Linear analysis results

\section{B. Simulation-based assessment results}

The controller is further validated in a nonlinear model of the aircraft including detailed nonlinear models of the aircraft aerodynamics, the actuators and the sensors. The model itself is a blackbox running on a Linux machine and has an interconnection interface to Windows and Matlab/Simulink. ${ }^{5}$ This enables to replace the nominal controller with the fault tolerant backup law developed in this paper. A negative step input on the pilot stick is used to test the response of the augmented aircraft.
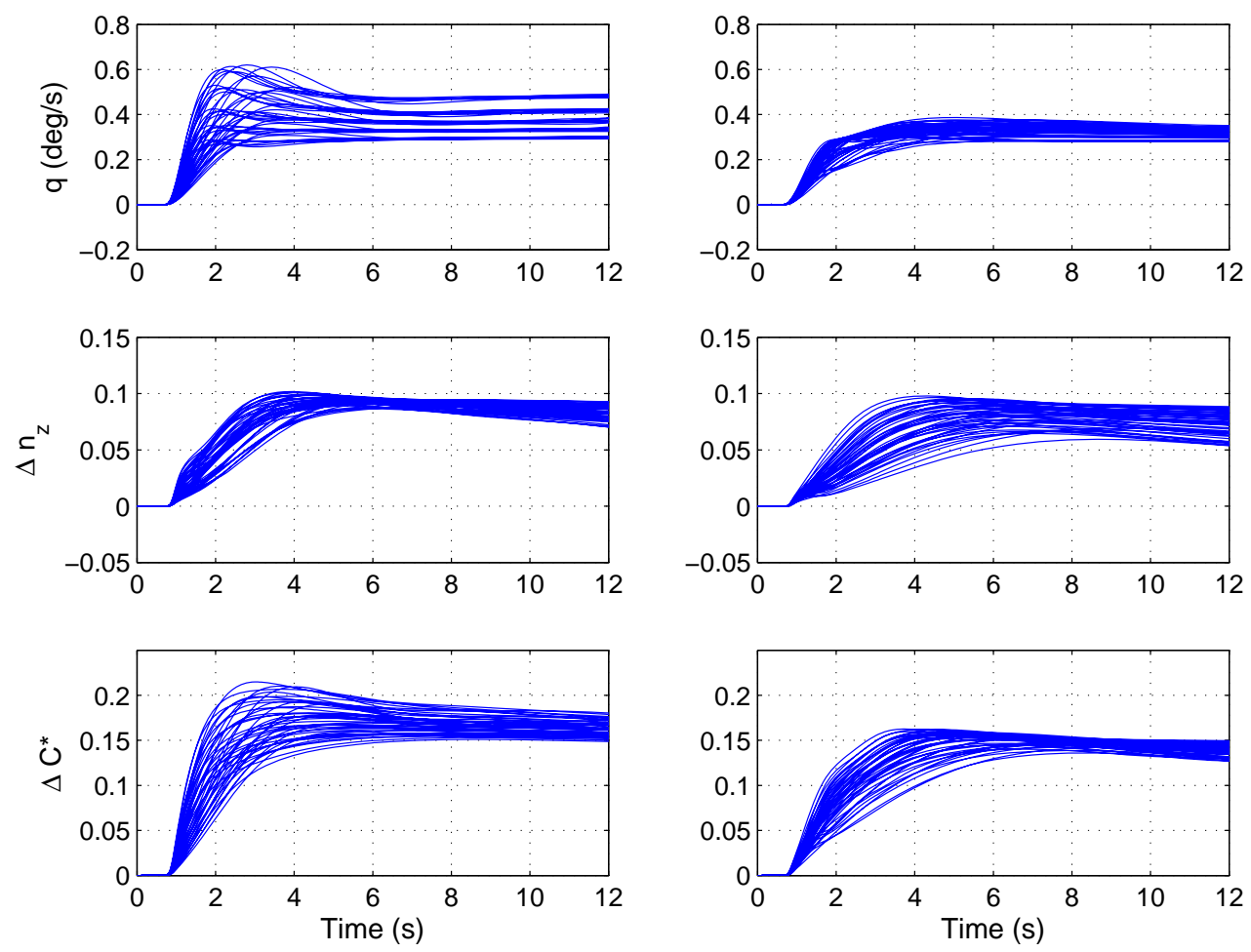

Figure 12. Simulated step responses of the fully scheduled nominal controller (left diagrams) and the backup controller (right diagrams) 
Figure 12 shows the simulation results for the 44 trim points of the first envelope region. Compared to the results of the nominal controller depicted in the left diagrams the overshoot in the load factor and in the pitch rate of the backup controller is lower, resulting in an increase of the settling time. Further it can be noticed that due to the $C^{*}$ command signal the spread in the final value of the load factor is higher for the backup controller compared to the nominal controller which uses a pure load factor command law. Although the responses of the system augmented with the backup controller are slower than the ones of the fully scheduled controller, the results stay close to the limits of the design requirements and thereby ensure good flying qualities.

This is confirmed when looking at the worst case results for the five envelope regions listed in table 2 determined by simulating the aircraft model on all 214 points. The overshoot values of the pitch rate and load factor lie below the ones of the fully scheduled controller, while the maximum values of the load factor settling time show a small increase compared to the nominal controller. This is an expected result, as during the design process the goal was to lie as close as possible to the lower tracking bound when shaping the frequency responses, thereby lowering the systems' bandwidths. However, a backup controller providing slightly slower responses than the nominal controller due to the lack of air data based scheduling variables is acceptable. Note that the settling times roughly correspond to the ones of the linear analysis, while the values in the overshoots are higher. This comes from the fact, that the values of the overshoot cannot be determined directly due to the phygoid dynamics, as there are no stationary values for load factor and pitch rate. This fact can also be identified in the pitch rate and load factor times responses depicted in figure 12. The stationary values are approximated by mean values over time, which are then used to determine the overshoots. To summarize the validation results, the backup controller introduces a small performance degradation of the closed loop behavior compared to the system augmented with the nominal, fully scheduled controller, however, still providing acceptable performance and stability characteristics.

\begin{tabular}{|l|c|c|c|c|c|c|}
\hline & \multicolumn{2}{|c|}{$q$ Overshoots (\%) } & \multicolumn{2}{c|}{$n_{z}$ Overshoots (\%) } & \multicolumn{2}{c|}{$n_{z}$-Settling times (sec) } \\
& Nominal & Backup & Nominal & Backup & Nominal & Backup \\
\hline Envelope region 1 & 53 & 32 & 27 & 18 & 5.7 & 6.5 \\
Envelope region 2 & 60 & 37 & 30 & 21 & 5.5 & 6.2 \\
Envelope region 3 & 53 & 22 & 13 & 7 & 6.0 & 6.6 \\
Envelope region 4 & 54 & 40 & 27 & 22 & 6.0 & 6.4 \\
Envelope region 5 & 75 & 13 & 32 & 9 & 6.3 & 6.4 \\
\hline
\end{tabular}

Table 2. Worst case values of the relevant design specification parameters determined by nonlinear simulations in the different flight regions

\section{Conclusion}

In this paper we demonstrated the successful design of a fault tolerant backup controller for modern civil aircraft using quantitative feedback theory. As the loss of scheduling information induced by a loss of the air data measurement system can degrade the performance of the aircraft, the backup controller needs to be designed with the goal to achieve the best performance and robustness using the remaining reliable measurements for feedback and scheduling. Using this approach, the way from a fully augmented aircraft to a controller with limited functionality or even a direct link law can be avoided in case of the loss of scheduling information. To provide a reasonable but alternative scheduling of the controller, flying quality criteria evaluations were used for the selection of useful scheduling variables. As a recommendation it can be stated that the selection and determination of the performance and stability design bounds for the backup law should be based on the specifications of the nominal controller. This will ensure a closed loop behavior as close as possible to the nominal one also in case of faults. 


\section{Acknowledgment}

This work was performed in the framework of the European FP7 RECONFIGURE Project: Grant agreement AAT-2012-RTD-2314544.

\section{References}

${ }^{1}$ Puyou, G. and Losser, Y., "Clearance Benchmark for a Civil Aircraft," Optimization Based Clearance of Flight Control Laws, edited by A. Varga, A. Hansson, and G. Puyou, Springer, Berlin, 2012, pp. 11-38.

${ }^{2}$ Oudin, S., Ferreres, G., Puyou, G., and Mouyon, P., "Adaptive LFT control of a transport aircraft on the lateral axis," Proc. of the IEEE Multi-Conference on Systems and Control (CCA Part), 2012.

${ }^{3}$ Varga, A., Ossmann, D., and Joos, H.-D., "A fault diagnosis based reconfigurable longitudinal control system for managing loss of air data sensors for a civil aircraft," Proc. of 18th IFAC World Congress, 2014.

${ }^{4}$ Ossmann, D. and Varga, A., "Optimization-based tuning of LPV fault detection filters for civil transport aircraft," Proc. of 4 th European Conference for Aerospace Sciences, St. Petersburg, Russia, 2011.

${ }^{5}$ Goupil, P., Boada-Bauxell, J., Marcos, A., Cortet, E., Kerr, M., and Costa, H., "AIRBUS efforts towards advanced real-time Fault Diagnosis and Fault Tolerant Control," Proc. of 19th IFAC World Congress, 2014.

${ }^{6}$ Horowitz, I. M., "Quantitative Feedback Design Theory (QFT)," QFT Publications, Boulder, Colorado, 1993.

${ }^{7}$ Doyle, J., "Qunatitative Feedback Therory (QFT) and Robust Control," Proc. of American Control Conference, 1986.

${ }^{8}$ Houpis, C. H. and Rasmussen, S. J., Quantitative Feedback Theory - Findamentals and Applications, Marcel, 1999.

${ }^{9}$ Nandakumar, R., Halikias, G., and Zolotas, A., "An Optimization algorithm for designing fixed structure controllers using the QFT method," Proc. of the 2002 IEEE Int. Symposium on Computer Aided Control System Design, 2002.

${ }^{10}$ Hodgkinson, J., Aircaft Handling Qualities, Blackwell Science, 1999.

${ }^{11}$ Schuck, F., Heller, M., Baier, T., and Holzapfel, F., "Longitudinal Robust Controller for Excellent Handling Qualities Design of a Gernal Aviation Aircraft using QFT," Proc. of AIAA Guidance, Navigation and Control Conference, 2013.

${ }^{12}$ Skogestad, S. and Postlethwaite, I., Multivariable Feedback Control - Analysis and Design, John Wiley \& Sons, 2005.

${ }^{13}$ Field, E., "The aplication of a $\mathrm{C}^{*}$ flight control law to large civil transport aircraft," Tech. rep., College of Aeronautics, Cranfield Institute of Technology, 1993.

14 "MIL-F-8785C, Military Specification, Flying Qualities of Piloted Airplanes," 1980.

${ }^{15}$ Odell, R., Patcher, M., and Hopkins, C. H., "Full Envelope Flight Control System Design Using Quantitative Feedback Theory," Journal of Guidance, Control, and Dynamics, Vol. 19, 1996, pp. 23-29.

${ }^{16}$ Brockhaus, R., Alles, W., and Luckner, R., Flugregelung, Springer Verlag Berlin Heidelberg, 3rd ed., 2011. 